\title{
INVASIVE GROUP A STREPTOCOCCAL INFECTIONS IN ONTARIO, CANADA
}

\author{
H. Dele Davies, M.D., Allison McGeer, M.D., Benjamin Schwartz, M.D., Karen Green, R.N., Darlene Cann, R.N., \\ Andrew E. Simor, M.D., Donald E. Low, M.D., and the Ontario Group A Streptococcal Study Group*
}

\begin{abstract}
Background Several reports suggest that the incidence of invasive group A streptococcal infections, including streptococcal toxic shock syndrome and necrotizing fasciitis, is increasing.

Methods During 1992 and 1993 we conducted prospective, population-based surveillance of invasive group A streptococcal disease in Ontario, Canada. We reviewed clinical and laboratory records, searched for secondary cases of invasive disease, and cultured specimens from household contacts.

Results We identified 323 patients with invasive group A streptococcal infections, for an annual incidence of 1.5 cases per 100,000 population. The rates were highest in young children and the elderly. Fiftysix percent of the patients had underlying chronic illness. Risk factors for disease included infection with the human immunodeficiency virus, cancer, diabetes, alcohol abuse, and chickenpox. The most common clinical presentations were soft-tissue infection (48 percent), bacteremia with no septic focus (14 percent), and pneumonia (11 percent). Necrotizing fasciitis occurred in 6 percent of patients, and toxic shock in 13 percent. The mortality rate was 15 percent overall, but it was 29 percent among those over 64 years of age $(P<0.001)$ and 81 percent among those with toxic shock $(\mathrm{P}<0.001)$. Fourteen percent of the cases were nosocomial, and 4 percent occurred in nursing home residents, often in association with disease outbreaks. Invasive disease occurred in 2 household contacts of patients with infection, for an estimated risk of 3.2 per 1000 household contacts (95 percent confidence interval, 0.39 to 12 per 1000).

Conclusions The elderly and those with underlying medical conditions are at greatest risk for invasive group A streptococcal disease, toxic shock, and necrotizing fasciitis. Invasive streptococcal infection is associated with a substantial risk of transmission in households and health care institutions. (N Engl J Med 1996;335:547-54.)

(C)1996, Massachusetts Medical Society.
\end{abstract}

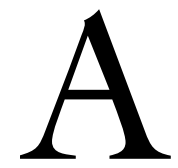

FTER several decades of steadily declining morbidity and mortality from group A streptococcal infections, the past 10 years have witnessed a striking resurgence in reports of severe disease, including the streptococcal toxic shock syndrome and necrotizing fasciitis. ${ }^{1-10}$ An understanding of the changes in the epidemiology of invasive group A streptococcal infections is important for optimal diagnosis and therapy and for the development of preventive strategies. Although several hospital-based case series have been reported, their limited size and potential bias due to referral patterns limit the generalizability of the findings. Only one population-based, retrospective study has been reported, ${ }^{9}$ and no prospective studies. $\mathrm{Al}-$ though reference laboratories in the United States, the United Kingdom, and Sweden have reported shifts in the serotypes of isolates to a greater frequency of $\mathrm{M}$ serotypes associated with greater virulence, ${ }^{3,6,9,11,12}$ no population-based data are available.

Recent reports have described clusters of invasive group A streptococcal infection, including the streptococcal toxic shock syndrome, in households, nursing homes, and hospitals. ${ }^{5}$ These descriptions have led some authorities to suggest that close contacts of persons with the streptococcal toxic shock syndrome should receive antibiotic therapy to eradicate group A streptococci, if present. ${ }^{13}$ However, the rate of secondary disease is unknown. The objectives of this study were to obtain population-based data on the incidence of invasive group A streptococcal disease, to define risk factors and clinical characteristics, and to assess the rate of secondary disease.

\section{METHODS}

\section{Population-Based Surveillance}

From January 1, 1992, to December 31, 1993, we studied all invasive group A streptococcal infections in persons living in Ontario, Canada (population, 10.7 million; 298,441 live births in 1992-1993). Invasive group A streptococcal disease was defined by the isolation of Streptococcus pyogenes from a normally sterile body site. Persons with invasive disease were considered to have the streptococcal toxic shock syndrome if the case met the consensus definition - that is, hypotension in combination with at least two of the following: acute renal failure, coagulation or liver abnormalities, rash, and necrotizing fasciitis. ${ }^{10}$ Patients who were dead on arrival at the hospital or who died within 48 hours of the onset of illness with insufficient laboratory data available for classification were also considered to have had the streptococcal toxic shock syndrome. Cases were considered nosocomial if the disease was not present or incubating at the time of admission. ${ }^{14}$

All 155 microbiology laboratories serving Ontario hospitals

From the Division of Infectious Diseases, Hospital for Sick Children (H.D.D.), the Department of Microbiology, Mount Sinai and Princess Margaret Hospitals (A.M., K.G., D.C., D.E.L.), and the Department of Microbiology, Sunnybrook Health Sciences Centre, University of Toronto (A.E.S.) - all in Toronto; and the Centers for Disease Control and Prevention, Atlanta (B.S.). Address reprint requests to Dr. Low at the Department of Microbiology, Mount Sinai Hospital, 600 University Ave., Toronto, ON M5G 1X5, Canada.

*The other members of the Ontario Group A Streptococcal Study Group are listed in the Appendix. 
and the largest laboratory serving physicians' offices telephoned the study office whenever $S$. pyogenes was isolated from specimens other than throat or eye swabs. We contacted attending physicians to identify isolates from sterile sites, to confirm that the patient was an Ontario resident, and to collect basic data (age, sex, infection site, and outcome). Informed consent was obtained to collect isolates and detailed clinical data. Annual audits of sterile-site cultures from all laboratories were performed to evaluate the accuracy of reporting. The study was approved by the Human Subjects Review Committee of the University of Toronto.

We attempted to classify cases into the same chronic-disease categories used by the Ontario Health Survey. ${ }^{15}$ Combining the prevalence data from the Ontario Health Survey and the category-specific numbers of cases from our own survey, we estimated category-specific rates of incidence and relative incidence. In calculating the confidence intervals for these estimates, we ignored sampling variation in the population estimates from the Ontario Health Survey, which was much smaller than the statistical error in the case counts. The estimated prevalence of human immunodeficiency virus (HIV) infection was obtained from the Ontario Ministry of Health (Wallace E: personal communication). We calculated the attack rate associated with cases of chickenpox for children under 10 years of age, using as the numerator the number of cases complicating an acute attack of chickenpox and estimating the denominator (the number of cases of chickenpox in Ontario over the two-year period) by assuming that 85 percent of the population is infected by age 10 , that the incidence is constant over time, and that 95 percent of the infections are associated with skin lesions. ${ }^{16}$ Household size was estimated from data obtained directly from the patients.

\section{Investigation of Disease Transmission}

From January 1, 1992, to April 1, 1993, patients with invasive disease who lived within 40 miles of the study office and all patients with the streptococcal toxic shock syndrome whose physicians agreed were asked for permission to approach their household contacts. Consenting household contacts were interviewed regarding the degree of contact with the patient and recent infections; throat and skin-lesion swabs were obtained to screen for $S$. pyogenes.

When more than one case of invasive disease was reported from the same or a nearby hospital or among residents of the same geographic area within a one-month period, the patients were questioned regarding possible contact. Links were also sought between patients from the same geographic area whose infections were caused by the same $\mathrm{M}$ or $\mathrm{T}$ serotype and occurred within three months of one another. For the purposes of this study, a subsequent, related case of invasive group A streptococcal infection was defined as one caused by the same strain as another case (see Laboratory Methods), with a clear epidemiologic link between the two cases.

When invasive infection was identified in a resident of a longterm care facility for the elderly, that facility was asked to collaborate in further investigations. Investigations consisted of chart reviews, questionnaires administered to staff members, and cultures of swabs from the throats and any skin lesions of consenting staff members and all residents. Where outbreaks were identified, definite $S$. pyogenes infection was diagnosed on the basis of culture-confirmed cellulitis, pharyngitis, pneumonia, or conjunctivitis, and possible $S$. pyogenes infection was diagnosed on the basis of cellulitis or pharyngitis that had not been confirmed by culture.

\section{Laboratory Methods}

Isolates were confirmed as $S$. pyogenes with the use of standard techniques. Typing on the basis of $\mathrm{M}$ precipitation and $\mathrm{T}$ agglutination was performed at the National Centre for Streptococcus, Edmonton, Alberta. ${ }^{17-19}$ Pulsed-field gel electrophoresis (PFGE) was performed with SmaI. ${ }^{20}$ Isolates were defined as the same strain if they were of the same $M$ and $T$ serotype and the restriction-fragment pattern on PFGE did not differ by more than one band. The presence of $s p e \mathrm{~A}$ and $s p e \mathrm{C}$ genes was assessed with the polymerase chain reaction. ${ }^{21}$

\section{Statistical Analysis}

Differences in group proportions were assessed by the chisquare test or Fisher's exact test. Differences in means were assessed by Student's t-test (for normally distributed variables) or the Wilcoxon rank-sum test. Differences in the incidence of disease among groups were assessed with a normal approximation for the comparison of Poisson counts. ${ }^{22}$ Logistic-regression models were used to assess risk factors for streptococcal toxic shock syndrome and for colonization of household contacts. Exact 95 percent confidence limits for the risk of disease in household contacts were determined with use of the binomial distribution.

\section{RESULTS}

During the study period, 323 cases of invasive group A streptococcal disease were identified: 298 (92 percent) were reported at the time of infection, and 25 ( 8 percent) were detected by laboratory audit. No sterile-site isolates were from specimens collected in physicians' offices. Basic data were available for all patients; isolates and detailed clinical data were obtained from 255 consenting patients ( 79 percent).

The incidence of invasive group A streptococcal disease was 1.5 per 100,000 population per year (1.3 per 100,000 in 1992 , as compared with 1.7 per 100,000 in $1993 ; \mathrm{P}=0.14$ ) (Fig. 1). The median age of the patients was 41 years (range, 2 weeks to 97 years); children and the elderly were at the highest risk for infection (Fig. 2). The higher-thanexpected number of infections among patients 30 to 39 years of age was due mainly to puerperal sepsis among the women and arthritis among the men. The rates in male and female patients were not significantly different (1.6 and 1.4 per 100,000 , respectively; $\mathrm{P}=0.16)$. Several groups were identified as being at significantly increased risk for invasive group A streptococcal infection (Table 1 ). The attack rate for invasive disease associated with chickenpox in children less than 10 years of age was 4.4 per 100,000 cases. If one assumes that the risk associated with chickenpox is present for two weeks after the onset of lesions (the time required for all lesions to heal fully), then the estimated relative risk of invasive group A streptococcal disease during this period is 39 (95 percent confidence interval, 16 to 90 ; $\mathrm{P}<0.001$ ).

\section{Clinical Presentation}

The most common clinical presentations were skin or soft-tissue infection (48 percent), bacteremia with no septic focus (14 percent), and pneumonia (11 percent). Twenty of the 156 patients with soft-tissue infections had necrotizing fasciitis (13 percent). The clinical presentation varied significantly with age (Table 2). Upper respiratory tract foci were more common in children, arthritis and pelvic infections more common in young adults, and necrotizing fasciitis more common in the elderly. The proportion of pa- 


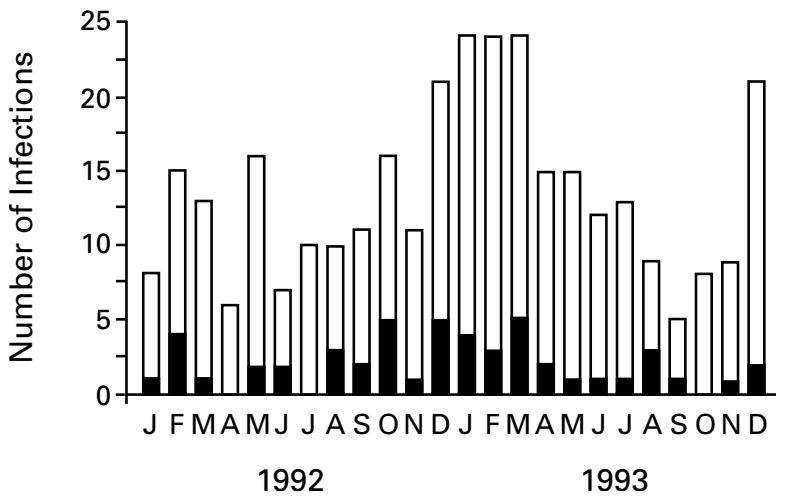

Figure 1. Occurrence of Invasive Group A Streptococcal Infections in Ontario in 1992 and 1993.

The solid portion of each bar represents cases associated with the streptococcal toxic shock syndrome.

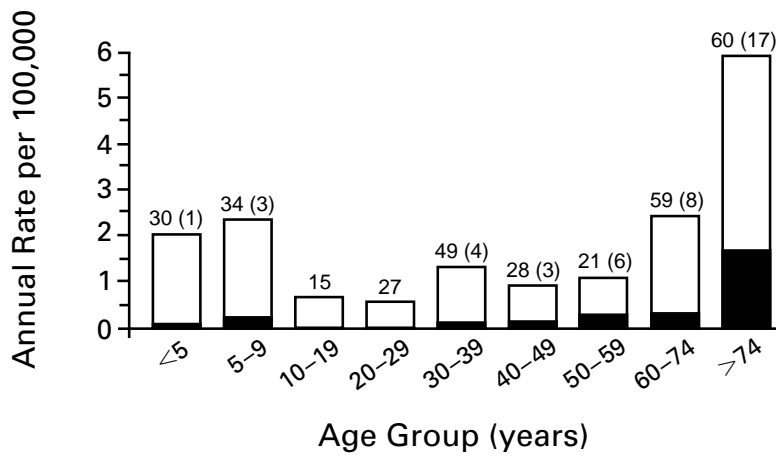

Figure 2. Age-Specific Rates of Invasive Group A Streptococcal Infection Occurring in Ontario in 1992 and 1993.

For each category the total number of cases is given above the bar, with the number of cases of the streptococcal toxic shock syndrome given in parentheses. The solid portion of each bar represents cases associated with the streptococcal toxic shock syndrome. The rates of invasive disease were highest in those less than 10 and older than 59 years of age $(P<0.001)$, and the rates were greater in those 30 to 39 years of age than in those who were 20 to 29 or 40 to 49 years old $(P=0.02)$. Four of 73 invasive cases ( 5 percent) in children up to 14 years old were associated with the streptococcal toxic shock syndrome, as compared with 6 of 97 in patients 15 to 44 years old (6 percent), 15 of 87 in those 45 to 74 years old (17 percent), and 17 of 59 in those 75 and older (29 percent) $(P<0.001)$. For seven patients there was insufficient information for the case definition to be applied; those patients have been excluded from this figure.

tients with chronic illness was higher among those with necrotizing fasciitis and pneumonia and lower among patients with pelvic infections. The streptococcal toxic shock syndrome and death were most commonly associated with the diagnoses of necrotizing fasciitis and pneumonia (Table 2).

Of the 44 nosocomial infections (14 percent of the total), 16 occurred in women post partum (14 cases of endometritis after vaginal delivery and 2 surgical-site infections after cesarean section), for a rate of 0.5 infection per 10,000 deliveries. The remaining 28 cases included 15 surgical-site infections, 8 cases of primary bacteremia ( 6 associated with intravenous access), 4 soft-tissue infections, and 1 case of pneumonia. Fourteen patients ( 4 percent) acquired their infections in residential long-term care facilities for the elderly.

Forty-two patients (13 percent) were classified as having toxic shock (annual rate, 0.2 per 100,000 population): 31 fulfilled the consensus definition of the syndrome, 4 were dead on arrival to the hospital, and 7 died shortly after admission without having sufficient information available for classification. The patients who had the streptococcal toxic shock syndrome were older than the other patients (median age, 61 , as compared with $38 ; \mathrm{P}<0.001$ ), more likely to have an underlying chronic illness (71 percent, as compared with 51 percent; $\mathrm{P}=0.03)$, and less likely to have nosocomial disease ( 2 percent, as compared with 15 percent; $\mathrm{P}=0.02$ ). These three associations remained significant when the analysis included only cases of the streptococcal toxic shock syndrome that met the consensus criteria.

Forty-two patients (13 percent) died within seven days of the onset of illness; an additional eight ( 2 percent) died later during hospitalization. The case fatality rate was higher in the elderly (29 percent for those 65 years of age or older, as compared with 8.3 percent for children up to 14 and 10 percent for persons 15 to 64 years of age; $\mathrm{P}<0.001$ by the chi-square test for trend) and in those with a chronic underlying illness (33 of 136 , as compared with 5 of $115 ; \mathrm{P}<0.001$ ). The overall mortality rate was 81 percent among patients with the streptococcal toxic shock syndrome (65 percent among those whose illness met the consensus definition), as compared with 5 percent ( 15 of $277)$ among those without the syndrome $(\mathrm{P}<0.001)$.

\section{Microbiologic Findings}

The majority of patients $(222$, or 69 percent $)$ had bacteremia. Among the 101 patients without bacteremia, S. pyogenes was isolated from intraoperative specimens (50 patients), synovial fluid (31), pleural fluid (6), cerebrospinal fluid (2), tissue obtained at autopsy (3), and other aspirates (9). Only 3 of 41 patients (7 percent) with the streptococcal toxic shock syndrome who had blood specimens cultured had negative results. These three patients were the only ones with negative blood cultures who died. Seven of 20 patients with necrotizing fasciitis ( 35 percent) had negative blood cultures.

Of the 258 isolates available (representing 80 percent of the total patient group), the most common serotypes were Ml (24 percent), Ml2 (7.4 percent), M4 (6.5 percent), M28 (6.2 percent), and M3 (5.8 percent). The proportion of cases caused by $\mathrm{Ml}$ 
Table 1. Risk of Invasive Group a Streptococcal Infection Associated with Selected Underlying Conditions.

\begin{tabular}{|c|c|c|c|c|c|}
\hline $\begin{array}{l}\text { UNDERLYING } \\
\text { CondITION }\end{array}$ & $\begin{array}{c}\text { AGE } \\
\text { GROUP }\end{array}$ & $\begin{array}{c}\text { No. of } \\
\text { CASES/TotAL } \\
(\%)^{*}\end{array}$ & $\begin{array}{c}\text { ANNUAL } \\
\text { INCIDENCE } \\
\text { (PER 100,000) }\end{array}$ & $\begin{array}{l}\text { Relative } \\
\text { RISK } \\
(95 \% \mathrm{CI}) \dagger\end{array}$ & $\begin{array}{c}\mathbf{P} \\
\text { VALUe }\end{array}$ \\
\hline HIV infection & $20-60 \mathrm{yr}$ & $4 / 97 \quad(4)$ & 10 & $9.4(3.5-25)$ & 0.001 \\
\hline Cancer & All & $24 / 251(10)$ & 7.5 & $6.9(4.5-10)$ & $<0.001$ \\
\hline Heart disease & All & $40 / 251(16)$ & 8.5 & $8.4(6.0-12)$ & $<0.001$ \\
\hline Diabetes & All & $21 / 251(8)$ & 4.1 & $3.7(2.4-5.8)$ & $<0.001$ \\
\hline Lung disease & All & $23 / 251(9)$ & 3.2 & $2.8(2.0-4.4)$ & $<0.001$ \\
\hline Alcohol abuse & $>16 \mathrm{yr}$ & $22 / 197(11)$ & 3.0 & $1.9(1.3-3.0)$ & 0.002 \\
\hline
\end{tabular}

* Information regarding underlying conditions was available for 251 patients, of whom 197 were older than 16 years of age and 97 were 20 to 60 years of age.

†CI denotes confidence interval.

strains increased from 15 percent in 1992 to 32 percent in $1993(\mathrm{P}=0.001)$, coincident with an increased rate of invasive disease, but a decrease in the proportion of cases of the streptococcal toxic shock syndrome $(\mathrm{P}=0.05)$. Changes in the frequency of other serotypes over time were not significant.

Sixty-four of 249 isolates tested (26 percent) contained the speA gene, and 110 (44 percent) the $s p e \mathrm{C}$ gene; only 13 strains possessed both toxin genes. There was a strong association between the Ml serotype and the presence of the speA gene (51 of 64 vs. 10 of $185, \mathrm{P}<0.001)$. In univariate analysis, strains possessing the speA gene were more often associated with the streptococcal toxic shock syndrome (14 of 36 vs. 50 of $213, \mathrm{P}=0.06)$ and death ( 14 of 37 vs. 50 of $212, \mathrm{P}=0.10)$ than other strains. However, there was no association between these outcomes and either the Ml serotype or the presence of the $s p e \mathrm{C}$ gene. In a logistic-regression model that included age, the presence of underlying illness, the $\mathrm{M}$ serotype, and the presence of the gene for speA or speC as potential risk factors, age was the only significant risk factor $(\mathrm{P}<0.01)$.

\section{Colonization of Household Contacts}

Eighty-one patients were approached regarding the household-contact portion of the study. Nineteen ( 23 percent) had no household contacts, and in 16 cases (20 percent) the patient, physician, or contacts refused to take part in the study. The remaining 46 patients had 152 household contacts. We obtained specimens for culture from the contacts a median of six days after the onset of illness in the index patient (83 percent within two weeks). Eighteen of 152 household contacts ( 12 percent) had throat cultures positive for $S$. pyogenes, all of which were the same strain as that of the index patient. Six of these 18 (33 percent) had pharyngitis at the time of culture (onset of illness, 5 to 12 days after the onset in the index patient); none had cellulitis or invasive infections. In univariate analysis, household contacts were more likely to be colonized if they were younger $(\mathrm{P}=0.004)$ and if they had four or more hours of contact daily with the index patient $(\mathrm{P}=0.02)$ (Fig. $3)$. These were the only two variables significantly associated with positive cultures in a logistic-regression model that also included the interval from the onset of illness to contact testing, whether or not the index patient had the streptococcal toxic shock syndrome, and the age of the index patient.

\section{Subsequent, Related Infections}

Seven cases of invasive disease ( 2 percent) met the criteria for subsequent, related infections. Two of these occurred among an estimated 620 household contacts of patients (estimated disease rate among household contacts, 3.2 per 1000 ; 95 percent confidence interval, 0.39 to 12 per 1000$)$. The first pair of cases was in a husband and wife, in whom the onset of pneumonia was separated by three days, and the second pair was in two brothers, in whom the onset of prepatellar bursitis was separated by three weeks. Two other cases were the result of transmission in different acute care hospitals: one was associated with an outbreak of postpartum endometritis, and one was a bacteremic soft-tissue infection linked to a prior case in a patient through a colonized health care worker. Two cases were associated with nursing home outbreaks, and one occurred in a household contact of a worker at a sheltered workshop, in association with a case of invasive disease in another worker. Probable community transmission was identified in two other situations. First, an adult residing in the same small community as the brothers described above (estimated population, 1000) became infected with the same strain five weeks after the second brother became ill. Second, 6 of 10 cases of infection caused by M-nontypable, T-serotype 5/27/44 isolates, which 
Table 2. Clinical Features Associated with 323 Cases of Invasive Group A STREPTOCOCCAL INFECTION.

\begin{tabular}{|c|c|c|c|c|c|c|}
\hline \multirow[t]{2}{*}{ Diagnosis } & \multirow[t]{2}{*}{$\begin{array}{c}\text { No. of } \\
\text { Patients }(\%) *\end{array}$} & \multicolumn{2}{|c|}{ AgE } & \multirow[t]{2}{*}{$\begin{array}{l}\text { UNDERLYING } \\
\text { CONDITION }\end{array}$} & \multirow[t]{2}{*}{$\begin{array}{l}\text { STREPTOCOCCAL } \\
\text { TOXIC SHOCK } \\
\text { SYNDROME }\end{array}$} & \multirow[t]{3}{*}{ DEATH } \\
\hline & & MEDIAN & RANGE & & & \\
\hline & & \multicolumn{2}{|c|}{$\mathrm{yr}$} & \multicolumn{2}{|c|}{ no. of patients (\%) } & \\
\hline Total & $323(100)$ & 44 & $0-97$ & $136(54)$ & $42(13)$ & $50(15)$ \\
\hline $\begin{array}{l}\text { Soft-tissue infection } \\
\text { Necrotizing fasciitis } \\
\text { Other }\end{array}$ & $\begin{array}{c}20(6) \\
136(42)\end{array}$ & $\begin{array}{l}68 \\
45\end{array}$ & $\begin{array}{c}19-87 \ddagger \\
0-97\end{array}$ & $\begin{array}{l}15(79) \S \\
74(54)\end{array}$ & $\begin{array}{l}11(55) \ddagger \\
12(9)\end{array}$ & $\begin{array}{r}9(45) \ddagger \\
15(11)\end{array}$ \\
\hline $\begin{array}{l}\text { Respiratory tract infection } \mathbb{} \\
\text { Upper } \\
\text { Lower }\end{array}$ & $\begin{array}{l}15(5) \\
36(11)\end{array}$ & $\begin{array}{r}8 \\
55\end{array}$ & $\begin{array}{l}3-69 \ddagger \\
1-95\end{array}$ & $\begin{array}{c}5(45) \\
23(77) \mathbb{S}\end{array}$ & $\begin{array}{l}0 \\
9(26) \mathfrak{S}\end{array}$ & $\begin{array}{l}0 \\
12(33) \ddagger\end{array}$ \\
\hline Bacteremia (no septic focus) & $46(14)$ & 55 & $0-89$ & $19(58)$ & $7(16)$ & $11(24)$ \\
\hline Arthritis & $26(8)$ & 39 & $0-89$ & $9(39)$ & $2(8)$ & $2(8)$ \\
\hline Pelvic infection\| & $20(6)$ & 32 & $19-58 \$$ & $2(13) \ddagger$ & 0 & $0 \S$ \\
\hline Other** & $24(7)$ & 39 & $1-86$ & $8(47)$ & $1(4)$ & $1(4)$ \\
\hline
\end{tabular}

*Because of rounding, the percentages do not total 100 percent.

†Because information regarding underlying conditions was available for only 251 patients, the denominators for this column may not equal the total number of patients in the first column of values.

$\ddagger \mathrm{P}<0.01$ for the comparison with all other patients.

$\$ \mathrm{P}<0.05$ for the comparison with all other patients.

IUpper respiratory tract infections included pharyngitis in 6 patients, oropharyngeal abscess in 4 , sinusitis in 3 , tracheitis in 1 , and supraglottitis in 1 ; lower respiratory tract infections included pneumonia in 35 patients and primary empyema in 1.

$\|$ Pelvic infections included postpartum endometritis in 13 patients, salpingitis in 3 , postoperative endometritis in 3 , and pyometria in 1 .

** Other diagnoses included intraabdominal infections in 12 patients, osteomyelitis in 5 , meningitis in 3 , urinary tract infection in 2 , and endocarditis in 2 .

were indistinguishable on analysis with PFGE, occurred in homeless people living in one metropolitan area, 5 of whom reported using the same shelter.

Fourteen invasive group A streptococcal infections occurred in residents of 12 long-term care facilities for the elderly. In four of seven facilities that agreed to participate in further investigations, previously unidentified outbreaks due to $S$. pyogenes were occurring. In facilities with outbreaks, a median of 6 percent (range, 3.3 to 9.6 percent) of residents had had a group A streptococcal infection, most often cellulitis, in the three months preceding the index case, and multiple residents and staff members (median, 4.3 percent of those tested; range, 2.2 to 7.1 percent) were colonized with the same strain of $S$. pyogenes.

\section{DISCUSSION}

Prospective, population-based studies provide important information on incidence rates, risk groups, and the settings in which preventive measures may be appropriate. The incidence of invasive group A streptococcal infection in this study ( 1.5 cases per 100,000 population per year) is similar to those reported in Sweden 6 and Cambridge, England, ${ }^{4}$ but lower than the incidence of 4.4 per 100,000 population detected in Pima County, Arizona.9 The differences in rates between Ontario and Pima County may be explained by differences in the proportions of high-risk populations or in the distribution of streptococcal strains. Our data also differ from those for Pima County in that 90 percent of the patients identified in that study had bacteremia, whereas almost one third of the patients in Ontario did not. In our study, surveillance for bacteremia alone would have identified more than 90 percent of persons who died of group A streptococcal infection or who had the streptococcal toxic shock syndrome, but it would have missed 35 percent of the cases of necrotizing fasciitis.

In contrast to prior series in which healthy young adults were found to be at risk for the streptococcal toxic shock syndrome and necrotizing fasciitis, 2,7 those at greatest risk in our population were older and had underlying medical conditions. This difference may reflect the lower likelihood of ascertainment bias in population-based data. Conversely, the case definition for the streptococcal toxic shock syndrome may identify not only those who have classic "toxic shock" but also those who are debilitated and more likely to have multiorgan failure when subjected to the stress of sepsis. 

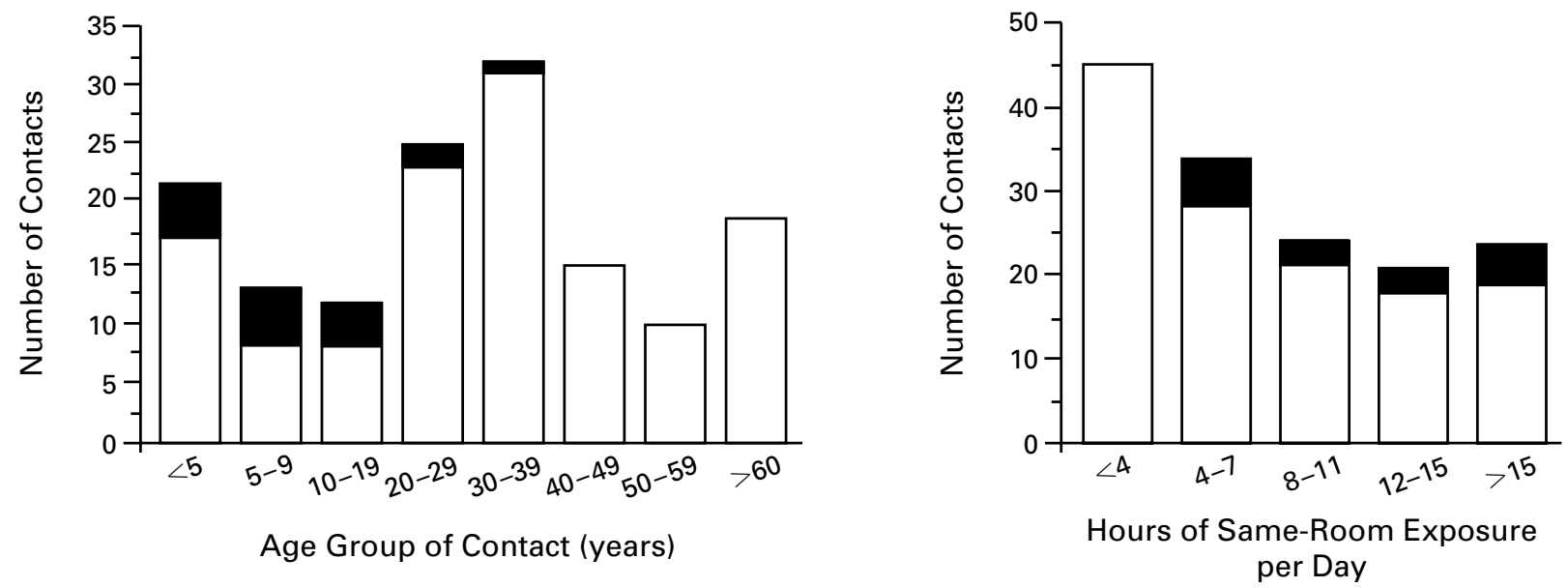

Figure 3. Risk Factors for Pharyngeal Infection or Colonization with the Same Strain of S. pyogenes in Household Contacts of Patients with Invasive Group A Streptococcal Infection.

The solid and open portions of the bars represent contacts with positive and negative pharyngeal cultures, respectively.

The magnitude of the increased risk of invasive group A streptococcal disease associated with lung and heart disease, cancer, and alcoholism should be interpreted with caution, since the data on cases were provided by physicians, whereas population data were based on personal interviews. Alcoholism was identified in patients by physicians but estimated for the entire population with a validated measure from questionnaires. ${ }^{15}$ Thus, the increase in the risk of disease associated with alcoholism is almost certainly low. The increase in risk associated with other chronic illnesses is similar to that reported for other bacterial infections and suggests that patients with these illnesses should be targeted if preventive measures such as immunization become available. This study also confirms suggestions from case series that the risk of invasive group A streptococcal disease is increased after chickenpox. ${ }^{23-25}$

The proportion of $\mathrm{Ml}$ isolates in our data (24 percent) is similar to that in data from the United Kingdom $^{4}$ and the United States. ${ }^{9,12}$ As has been observed elsewhere, $3,6,11,12,26$ we found that as the proportion of infections with the Ml serotype increased, the incidence of invasive disease also increased. In contrast to previous studies, ${ }^{1,2,6-9,12,27}$ however, we did not find an association between the Ml serotype or the presence of the speA gene and the streptococcal toxic shock syndrome after we controlled for age and the existence of underlying illness. Our inability to detect an association may reflect limitations in the power of the study or the lower predictive value of identifying the speA gene as compared with assaying for toxin production. ${ }^{28}$ Nonetheless, these data support the concept that other factors are important in the pathogenesis of the streptococcal toxic shock syndrome. ${ }^{29}$
Infections occurring in institutions or in contacts of patients provide a focus for preventive intervention. Surgical-site infections and puerperal sepsis accounted for a substantial portion of all invasive group A streptococcal infections. If organisms are acquired in the hospital, prevention depends on improving infection-control practices or identifying and treating staff members who are asymptomatic carriers. ${ }^{30-34}$ However, since some puerperal infections are most likely due to antenatal colonization, and since the rate of vaginal carriage of $S$. pyogenes late in pregnancy is very low, ${ }^{35}$ our data suggest that, if late prenatal screening for group B streptococci is being performed, identifying and reporting other $\beta$-hemolytic streptococci may also be useful.

Although outbreaks of group A streptococcal infections in nursing homes have been reported, 5,36,37 there are no data on their incidence or on the frequency with which invasive cases occur in the context of an outbreak within an institution. The insidious nature of outbreaks in this setting and the limited resources for surveillance in nursing homes may inhibit early recognition of outbreaks. ${ }^{37}$ Our investigation following a single case of invasive group A streptococcal disease led to the identification of unrecognized outbreaks in 4 of 7 facilities; this finding supports this hypothesis and suggests that any single case of invasive group A streptococcal infection in a nursing home demands further investigation.

Our study provides an estimate of the incidence of invasive disease among contacts of persons with invasive group A streptococcal disease. In continuing surveillance in Ontario, as of June 30, 1995, 2 additional cases of invasive infection had been identified in an additional 740 household members. 
Our best estimate of the risk to members of patients' households is thus 2.9 per 1000 (95 percent confidence interval, 0.80 to 7.5 per 1000 ), almost 200 times the risk in the general population. Although, in our study, no household contacts had the streptococcal toxic shock syndrome, such cases have been reported. $5,38,39$ The risk of disease among close contacts and the overall case fatality rate of invasive group A streptococcal infections are similar to those for sporadic meningococcal infections. ${ }^{5,39-41}$ These data support the use of chemoprophylaxis in patients' close contacts; however, the best approach to prophylaxis remains unclear.

Our data show that the risk of colonization in the household is associated with younger age and four or more hours of contact with the infected person per day, but the risk of disease is greatest in the elderly despite their lower carriage rates. Furthermore, it is usually not possible to determine whether invasive cases in two members of one household are the result of direct transmission from one to the other, indirect transmission through another colonized household member, or exposure of both household members to the same outside source. Until it is clear whether the primary goal of prophylaxis is to prevent disease in those recently colonized or to decrease transmission of a strain known to cause severe infection, it will not be possible either to recommend focusing prophylaxis on a subgroup of close contacts or to estimate the effectiveness of different approaches to prophylaxis.

Supported in part by a contract from the Centers for Disease Control and Prevention, Atlanta, a grant from MDS Health Group, Toronto, the Eli Lilly Canadian Infectious Diseases Fellowship Award (to Dr. Davies), and the Ontario Ministry of Health Research Personnel Development Award (to Dr. Davies)

We are indebted to the microbiology laboratories, infection-control practitioners, and physicians across Ontario, without whose time, effort, and enthusiasm this surveillance would not have been possible; to the many staff members of the Ontario Ministry of Health and public health departments across Ontario who have supported this study and collaborated in investigations; to the owners, staff members, and residents of the participating long-term care facilities; to Ruth Jaeger, Mark Jogersma, and Paul Grantham of MDS Health Group for the transport of specimens; and to Jeanette Huberty-Edwards, Alice Au Yeung, and Kim Wilson for their secretarial support.

\section{APPENDIX}

The following are other members of the Ontario Group A Streptococcal Study Group: A. Fletcher, R. Kaul, S. Scriver, and B. Willey (Mount Sinai and Princess Margaret Hospitals, Toronto); B. Demers (Institut Pasteur, Paris); W. Gold (Toronto Hospital, Toronto); M. Lovgren and J. Talbot (National Centre for Streptococcus, Edmonton, Alta.); and M. Naus (Ontario Ministry of Health, Toronto).

\section{REFERENCES}

1. Demers B, Simor AE, Vellend $H$, et al. Severe invasive group A streptococcal infections in Ontario, Canada: 1987-1991. Clin Infect Dis 1993;16: 792-800.

2. Cone LA, Woodard DR, Schlievert PM, Tomory GS. Clinical and bac teriologic observations of a toxic shock-like syndrome due to Streptococcus pyogenes. N Engl J Med 1987;317:146-9.

3. Holm SE, Norrby A, Bergholm AM, Norgren M. Aspects of pathogenesis of serious group A streptococcal infections in Sweden, 1988-1989.

J Infect Dis 1992;166:31-7.

4. Francis J, Warren R. Streptococcus pyogenes bacteraemia in Cambridge a review of 67 episodes. Q J Med 1988;68:603-13.

5. Schwartz B, Elliott JA, Butler JC, et al. Clusters of invasive group A streptococcal infections in family, hospital, and nursing home settings. Clin Infect Dis 1992;15:277-84.

6. Stromberg A, Romanus V, Burman LG. Outbreak of group A streptococcal bacteremia in Sweden: an epidemiologic and clinical study. J Infect Dis $1991 ; 164: 595-8$.

7. Stevens DL, Tanner MH, Winship J, et al. Severe group A streptococcal infections associated with a toxic shock-like syndrome and scarlet fever toxin A. N Engl J Med 1989;321:1-7.

8. Wheeler MC, Roe MH, Kaplan EL, Schlievert PM, Todd JK. Outbreak of group A streptococcus septicemia in children: clinical, epidemiologic, and microbiological correlates. JAMA 1991;266:533-7.

9. Hoge CW, Schwartz B, Talkington DF, Breiman RF, MacNeill EM, Eng lender SJ. The changing epidemiology of invasive group A streptococcal infections and the emergence of streptococcal toxic shock-like syndrome: a retrospective population-based study. JAMA 1993;269:384-9. [Erratum,

JAMA 1993;269:1638.]

10. The Working Group on Severe Streptococcal Infections. Defining the group A streptococcal toxic shock syndrome: rationale and consensus definitions. JAMA 1993;269:390-1.

11. Colman G, Tanna A, Efstratiou A, Gaworzewska ET. The serotypes of Streptococcus pyogenes present in Britain during 1980-1990 and their association with disease. J Med Microbiol 1993;39:165-78.

12. Schwartz B, Facklam RR, Breiman RF. Changing epidemiology of group A streptococcal infection in the USA. Lancet 1990;336:1167-71.

13. Group A streptococcal infections. In: 1994 Red book: report of the Committee on Infectious Diseases. 23rd ed. Elk Grove Village, Ill.: American Academy of Pediatrics, 1994:430-9.

14. Garner JS, Jarvis WR, Emori TG, Horan TC, Hughes JM. CDC definitions for nosocomial infections, 1988. Am J Infect Control 1988;16: 128-40. [Erratum, Am J Infect Control 1988;16:177.]

15. Premier's Council on Health, Well-Being and Social Justice. Highlights of the Ontario Health Survey, 1990. Toronto: Ontario Ministry of Health, 1992.

16. Preblud SR, Orenstein WA, Bart KJ. Varicella: clinical manifestations, epidemiology and health impact in children. Pediatr Infect Dis 1984;3: $505-9$.

17. Griffith F. Serological classification of Streptococcus pyogenes. J Hyg 1934;34:542-84.

18. Maxted WR, Widdowson JP, Fraser CA, Ball LC, Bassett DC. The use of the serum opacity reaction in the typing of group-A streptococci. J Med Microbiol 1973;6:83-90.

19. Rotta J, Krause RM, Lancefield RC, Everly W, Lackland H. New approaches for the laboratory recognition of $\mathrm{M}$ types of group A streptococci. J Exp Med 1971;134:1298-315.

20. Single LA, Martin DR. Clonal differences within M-types of the group A streptococcus revealed by pulsed field gel electrophoresis. FEMS Microbiol Lett 1992;70:85-9.

21. Tyler SD, Johnson WM, Huang JC, et al. Streptococcal erythrogenic toxin genes: detection by polymerase chain reaction and association with disease in strains isolated in Canada from 1940 to 1991. J Clin Microbiol 1992;30:3127-31.

22. Statistical inference. In: Armitage P, Berry G. Statistical methods in medical research. 2nd ed. Oxford, England: Blackwell Scientific Publications, 1987:134-7.

23. Davies HD, Matlow A, Scriver SR, et al. Apparent lower rates of streptococcal toxic shock syndrome and lower mortality in children with invasive group A streptococcal infections compared with adults. Pediatr Infect Dis J 1994;13:49-56.

24. Gonzalez-Ruiz A, Ridgway GL, Cohen SL, Hunt CP, McGrouther G, Adiseshiah M. Varicella gangrenosa with toxic shock-like syndrome due to group A streptococcus infection in an adult: case report. Clin Infect Dis 1995;20:1058-60.

25. Wilson GJ, Talkington DF, Gruber W, Edwards K, Dermody TS. Group A streptococcal necrotizing fasciitis following varicella in children: case reports and review. Clin Infect Dis 1995;20:1333-8.

26. Johnson DR, Stevens DL, Kaplan EL. Epidemiologic analysis of group 
A streptococcal serotypes associated with severe systemic infections, rheumatic fever, or uncomplicated pharyngitis. J Infect Dis 1992;166:374-82. 27. Cleary PP, Kaplan EL, Handley JP, et al. Clonal basis for resurgence of serious Streptococcus pyogenes disease in the 1980s. Lancet 1992;339:518-21. 28. Talkington DF, Schwartz B, Black CM, et al. Association of phenotypic and genotypic characteristics of invasive Streptococcus pyogenes isolates with clinical components of streptococcal toxic shock syndrome. Infect Immun 1993;61:3369-74

29. Watanabe-Ohnishi R, Low DE, McGeer A, et al. Selective depletion of $\mathrm{V} \beta$-bearing $\mathrm{T}$ cells in patients with severe invasive group A streptococcal infections and streptococcal toxic shock syndrome. J Infect Dis 1995;171: 74-84.

30. Schaffner W, Lefkowitz LB Jr, Goodman JS, Koening MG. Hospital outbreak of infections with group A streptococci traced to an asymptomatic anal carrier. N Engl J Med 1969;280:1224-5.

31. Stamm WE, Feeley JC, Facklam RR. Wound infections due to group A streptococcus traced to a vaginal carrier. J Infect Dis $1978 ; 138$ : 287-92.

32. Mastro TD, Farley TA, Elliott JA, et al. An outbreak of surgical-wound infections due to group A streptococcus carried on the scalp. N Engl J Med 1990;323:968-72

33. Paul SM, Genese C, Spitalny K. Postoperative group A $\beta$-hemolytic streptococcus outbreak with the pathogen traced to a member of a healthcare worker's household. Infect Control Hosp Epidemiol 1990;11:643-6.
34. Viglionese A, Nottebart VF, Bodman HA, Platt R. Recurrent group A streptococcal carriage in a health care worker associated with widely separated nosocomial outbreaks. Am J Med 1991;91:Suppl 3B:3B-329S-3B-333S 35. Bibler MR, Rouan GW. Cryptogenic group A streptococcal bacteremia: experience at an urban general hospital and review of the literature. Rev Infect Dis 1986;8:941-51.

36. Auerbach SB, Schwartz B, Williams DL, et al. Outbreak of invasive group A streptococcal infections in a nursing home: lessons on prevention and control. Arch Intern Med 1992;152:1017-22.

37. Schwartz B, Ussery XT. Group A streptococcal outbreaks in nursing homes. Infect Control Hosp Epidemiol 1992;13:742-7.

38. Dipersio JR, Define LA, Gardner W, Stevens DL, Kaplan EL, File TM. Use of pulsed field gel electrophoresis to investigate the clonal spread of severe group A streptococcal disease. In: Abstracts of the 95th General Meeting of the American Society for Microbiology, May 21-25, 1995.

Washington, D.C.: American Society for Microbiology, 1995:33. abstract. 39. Valenzuela TD, Hooton TM, Kaplan EL, Schlievert P. Transmission of 'toxic strep' syndrome from an infected child to a firefighter during CPR. Ann Emerg Med 1991;20:90-2.

40. The Meningococcal Disease Surveillance Group. Analysis of endemic meningococcal disease by serogroup and evaluation of chemoprophylaxis. J Infect Dis 1976;134:201-4.

41. Idem. Meningococcal disease: secondary attack rate and chemoprophylaxis in the United States, 1974. JAMA 1976;235:261-5. 